\title{
Functionally Expanded Phase-Change Memory: Experiments on Light Influence on Threshold Voltage
}

\author{
S. Asokan \\ Indian Institute of \\ Science, Bangalore, India
}

\author{
S. D. Savransky* \\ The TRIZ Experts, Newark, \\ California, USA
}

\author{
Pulok Pattanayak \\ Indian Institute of Science, \\ Bangalore, India
}

\author{
M. Anbarasu \\ Indian Institute of Science, \\ Bangalore, India
}

\begin{abstract}
We describe the first experimental results of a light influence on the threshold voltage $V_{t}$ in new ternary lead-free telluride compound (labeled as SA1). Reduction of $V_{t}$ on about $35 \%$ in SA1 illuminated by Ar ion laser to compare dark $V_{t}$ is discovered. More than 10,000 switching cycles without degradation have been recorded. Variation of the laser power allows achieving $V_{t}$ reduction in SA1 down to $40 \%$ from the dark level. This is the largest change of $V_{t}$ known for amorphous chalcogenides. It opens new horizons for chalcogenide functionally expanded phase change memory. Some ideas about the mechanism of the observed effect, related with the photo-generation of charge carriers and possible mechanisms of transition from OFF state to $\mathrm{ON}$ state, are discussed.
\end{abstract}

Keywords-telluride alloys; threshold switching; light influence; functionally expanded PC-RAM.

\section{INTRODUCTION}

Chalcogenide glasses are known to exhibit reversible (threshold) and irreversible (memory) switching from a semiconducting (high resistivity) OFF state to a conducting $\mathrm{ON}$ state $[1,2]$. The phenomenon of electrical switching in amorphous chalcogenides has many applications, which includes phase-change memory (PCRAM), solid state relay, sensors, restorable fuses, etc.

PC-RAM based on amorphous chalcogenides, which exhibit electrical switching, is considered as a promising replacement for existing nonvolatile memories [3]. One of most promising applications, namely the creation of hybrid optoelectronic memories, is generally limited by the non-sensitivity of the OFF-ON transition to light in traditional telluride alloys. Usually light does not influence on threshold voltage $\left(\mathrm{V}_{\mathrm{t}}\right)$ at all or the observed effect $[1,4,5,6]$ too small to be consider useful.

\footnotetext{
* Corresponding author. The TRIZ Experts, 6015 Pepper Tree Ct., Newark, CA 94560, USA Email: chalcogenide_glasses@yahoo.com
}

This obstacle can be removed in some chalcogenide glasses [7,8]. Earlier experiments on light influence on $\mathrm{V}_{\mathrm{t}}$ with $\mathrm{Pb}-\mathrm{Ge}-\mathrm{Te}$ amorphous films allow us to achieve technically acceptable levels of $\mathrm{V}_{\mathrm{t}}$ alteration $[7,8]$. However, these lead alloys have a relatively small number of transitions between OFF and ON states (i.e., poor endurance). In the present study, we describe some interesting preliminary results on light influence on threshold voltage of few specially selected lead-free chalcogenide glasses, which exhibit threshold-switching behavior and relatively good endurance. Absence of photo degradation in these samples, in the photoconductivity measurements, also makes them ideal candidates for the present study.

\section{EXPERIMENTAL PROCEDURE}

The ternary chalcogenide glasses have been prepared by vacuum-sealed melt quenching method. Appropriate quantities of high purity (99.99\%) constituent elements are sealed in an evacuated quartz ampoule (at $10^{-5}$ Torr) and slowly heated in a horizontal rotary furnace. The ampoules are maintained at $900{ }^{\circ} \mathrm{C}$ and rotated continuously for about $24 \mathrm{~h}$ at $10 \mathrm{rpm}$ to ensure homogeneity of the melt. The ampoules are subsequently quenched in a bath of ice water and $\mathrm{NaOH}$ mixture to get bulk glassy samples. The amorphous nature of the quenched samples is confirmed by X-ray diffraction.

One ternary telluride glass composition (named temporarily SA1) was selected for this research ${ }^{1}$. The I - V characteristics of these glass has been studied using a Keithley source meter (model: 2410c). Sample polished to the required thickness is mounted in a holder made of brass, in between a flat-plate bottom electrode and a point-contact top electrode using a springloading mechanism. A constant current is passed through the sample and the voltage developed

1 Unfortunately, we cannot disclosure the chemical compositions of the alloys until we complete the patent applications for the compounds and effect. 
across the sample, and the corresponding current through the sample, are measured.

To study the influence of illumination on the threshold voltage, Ar ion laser (model: $177 \mathrm{G} 42$, wavelength of $514 \mathrm{~nm}$ ) with variable power is used to shine the light on the sample. A mechanical chopper with variable frequency is placed in between the laser source and the sample.

\section{EXPERIMENTAL RESULTS}

The I - V characteristics of the SA1 compound studied, is shown in figure 1. It is seen from figure 1 that above a threshold voltage $\left(V_{t}\right)$, the sample exhibits a current-controlled negative resistance behavior, which leads to a lowresistance ON state. Further, the sample is found to revert back to the high-resistance OFF state upon reducing the current (threshold switching behavior [1]).

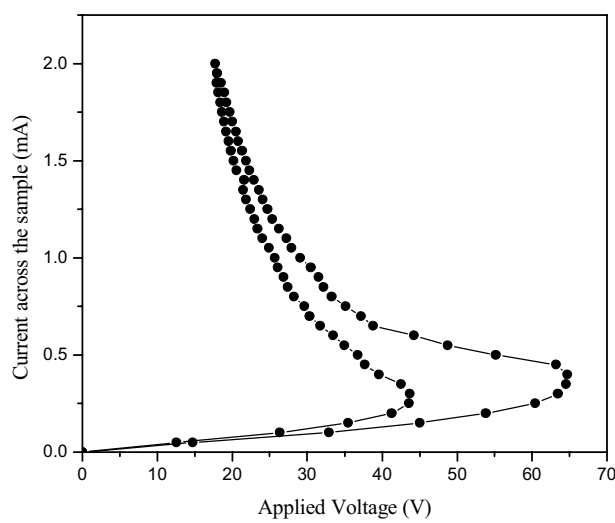

Figure 1. The I-V characteristics of SA1 alloy studied in dark. $\mathrm{V}_{\mathrm{t}} \sim 65 \mathrm{~V}$

The same sample is switched under illumination. In the present experiments, the chopping frequency and the laser power are kept constant at $0.5 \mathrm{~Hz}$ and $110 \mathrm{~mW}$ respectively. It can be seen from figure 2 that the threshold voltage reduces by approximately $35 \%$.

The reduction of $V_{t}$ depends upon the laser power and the percentage of reduction decreases with decrease in laser power. The reduction in switching voltages under illumination is essentially due to the creation of additional charge carriers by the incident light.

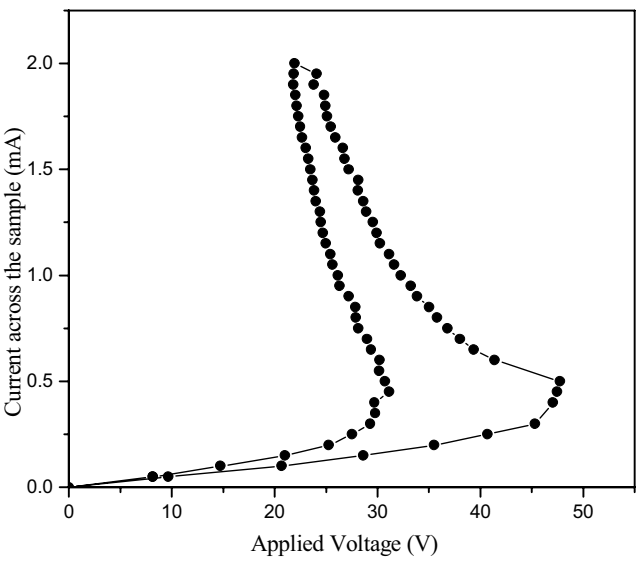

Figure 2. The I-V characteristics of the same SA1 sample under illumination. $\mathrm{V}_{\mathrm{t}} \sim 48 \mathrm{~V}$

It is also interesting to note that for a given laser power, the first switching cycle requires higher voltages than the subsequent cycles and the switching voltages stabilize after 3-4 cycles. The sample can be repeatedly switched subsequently and nearly 10,000 switching cycles have been carried out on the sample without any decrease in the switching voltages.

It has been found that the opto-electronic switching mainly depends upon four parameters, namely the voltage across the sample, the maximum on-state current flowing through the sample, chopping frequency and the laser power. Further, it has been found that a minimum of 0.4 $\left(V_{t}\right)$ has to be applied across the sample to induce the switching optically. This is the largest change of $\mathrm{Vt}$ known for amorphous chalcogenides [6-8]. Applying lower voltages across the sample leads only to the photoconductivity response of the sample and application of higher voltages latches the sample permanently into the ON state. In the present experiments, the voltage across the sample is maintained optimally at $0.7^{*} \mathrm{~V}_{\mathrm{t}}$. Similarly the current flowing through the sample in the $\mathrm{ON}$ state is restricted to $2 \mathrm{~mA}$, beyond which the sample latches permanently to the high conduction state.

Figure 3 shows the repeated transition exhibited by the sample from the OFF to the ON state induced by chopped laser light. More than 10,000 switching cycles ${ }^{2}$ have been observed in the present study if SET current is limited to 0.8 $\mathrm{mA}$, which indicates clearly the suitability of the studied SA1 glass for practical applications, e.g.

\footnotetext{
${ }^{2}$ We stopped experiments at this point, probably SA1
} has even better endurance. 
for the functionally expanded phase change memories.

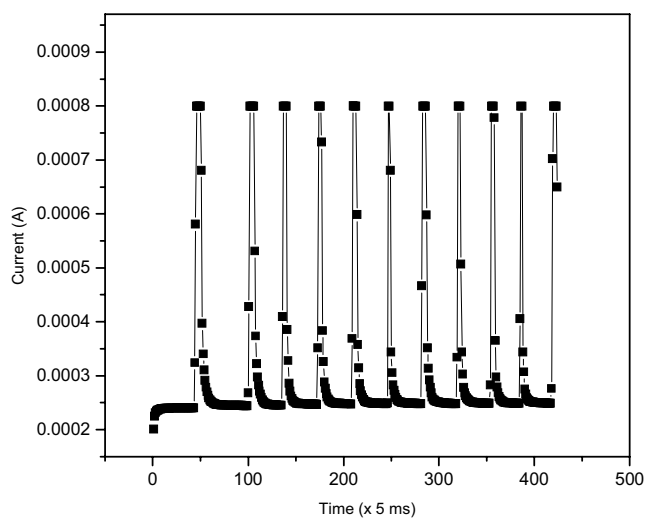

Figure 3. Repeated switching in SA1 samples induced optically

\section{DISCUSSION}

Threshold switching in typical chalcogenide glasses and their amorphous films is considered to be mainly electronic in nature $[1,2]$. Such OFF-ON transition occurs when the charged $-\mathrm{U}$ centers (often named as defect states $\mathrm{D}^{+}$and $\mathrm{D}^{-}$ or $\mathrm{C}^{+}$and $\mathrm{C}^{-}$) present in the material $[9,2]$ are compensated by the charge carriers excited by the electrical field $[1,10,11]$. Therefore, the switching effect in the previously studied chalcogenide glasses $[1,2,4-6,10,11]$ is driven by electrical fields (voltage). The reduction in switching voltages under illumination in $\mathrm{Pb}-\mathrm{Ge}-$ Te and SA1 alloys is essentially due to the creation of additional charge carriers by the incident light, which help to passivate or destroy the $-\mathrm{U}$ centers at earlier applied voltages. We think that the threshold switching in this SA1 as well as in Ge-Sb-Te alloys commonly used in PC-RAM [3] is the current driven effect [12]. Probably the excess charge carriers generated by laser beam redistribute spectra and (or) density of localized states in the mobility gap of such ternary telluride alloys that lead to the transition form semiconducting to conducting state. More details of this model as well as further experimental results will be presented elsewhere.

\section{CONCLUSION}

The effect of optical illumination on the threshold voltage of a selected lead-free chalcogenide glass, which exhibits excellent threshold switching behavior, is demonstrated. The electro-optical switching effect is due to the enhancement in the charge carriers by the light and applied electric field. It has been found that four parameters, applied constant voltage, the on-state current flowing through the sample, chopping frequency and laser power, can be controlled to get the desired performance. The irradiation of the sample with $110 \mathrm{~mW}$ laser light brings down the threshold voltage by about $35 \%$ compared to the dark level. Further, switching in this sample can be done repeatedly (more than 10,000 cycles) using chopped light, while keeping the sample under constant voltage. The present results open up new horizons for chalcogenide non-volatile memory applications.

\section{REFERENCES}

[1] D. Adler, H. K. Heinz and N. Mott, "The mechanism of threshold switching in amorphous alloys," Reviews of Modern Physics, vol. 50, pp. 209-220, 1978.

[2] N. F Mott and E. A. Davis, Electronic Processes in Non-Crystalline Materials, $2^{\text {nd }}$ ed., Oxford: Clarendon, 1979, $590 \mathrm{pp}$

[3] S. Hudgens and B. Johnson, "Overview of PhaseChange Chalcogenide Nonvolatile Memory Technology," MRS Bulletin, vol. 29, pp. 829832,2004

[4] H. K. Henisch, R. W. Pryor, and G. J. Vendura, "Characteristics and mechanism of threshold switching," Journal of Non-Crystalline Solids, vol. 8-10, pp. 415-421, 1972.

[5] L. A. Coldren, M. A. Bösch, and J. A. Rentschler, "Multistate amorphous-semiconductor switch," Applied Physics Letters, vol. 36, pp. 688-690, 1980.

[6] S. A. Kostylev and V. A. Shkut, Electronic switching in the amorphous semiconductors, Kiev: Naukova Dumka, 1978. 203 pp. (in Russian).

[7] S.D. Savransky and S.E. Sofronev, "Peculiarities of the conductivity and switching effect in $\mathrm{Pb}$ Ge-Te glasses," Abstracts 3 USSR Conf. "Structure and Properties of Metal Melts" Sverdlovsk, vol. 2, p.248, 1986.

[8] S.D. Savransky, Photo-switching element. USSR Patent N 1342277. MCI G02F 1/29. Received August 20, 1984. Registered June 1, 1987

[9] P. W. Anderson "Model for the electronic structure of amorphous semiconductors," Physical Review Letters, vol.34, pp. 953-955, 1975.

[10] .D. Adler, M. S. Shur, M. Silver, and S. R. Ovshinsky, "Threshold switching in chalcogenide-glass thin films," Journal of Applied Physics, vol. 51, pp. 3289-3309, 1980.

[11] K. E. Petersen and David Adler, "On state of amorphous threshold switches," Journal of Applied Physics, vol. 47, pp. 256-263, 1976.

[12] S. D. Savransky, "Model of conductivity transition in amorphous chalcogenides induced by Auger recombination," Journal of Ovonic Research, vol. 1, pp.25-30, 2005. 